\title{
THE USE OF FERROMAGNETIC AMORPHOUS MATERIALS 5BDSR IN HIGH-FREQUENCY OVERVOLTAGE PROTECTION DEVICES
}

\author{
Sergey Korobeynikov and Valentin Loman \\ Power Engineering Faculty, Novosibirsk State Technical University, Novosibirsk, Russia, \\ e-mail: Loman_95mail.ru
}

Received Date: August 14, 2020; Revised Date: October 19, 2020; Acceptance Date: December 1, 2020

\begin{abstract}
The paper discusses the prospects of using nanocrystalline amorphous materials in the electric power industry. In particular, in the field of protection of electric power equipment of substations from high-frequency pulses. The effectiveness of using such materials to activate the anomalous skin effect in multilayer conductors is shown. The resulting conductors increase their resistance at lightning frequencies, thereby making it possible to create devices that effectively suppress highfrequency pulses. The authors give an example of a protective frequency-dependent device using nanocrystalline ferromagnetic material and the results of its use in trial operation. The paper shows the results of comparing round and flat frequency-dependent conductors.
\end{abstract}

Keywords: Amorphous material, Frequency dependent device (FDD), High frequency overvoltage, Nanocrystalline film, Very fast transient overvoltages (VFTO)

\section{Introduction}

Growth of technologies level and production allows the development of devices and components based on nanomaterials. Such materials have found their application in the electric power industry - this is especially evident in the example of amorphous and magnetically soft materials. Amorphous materials are used to manufacture transformer equipment, telecommunications systems, solar generators, switching power supplies, electromagnetic interference filters, various sensors, etc. [1] - [4]. It is important to note that the replacement of conventional transformer steel with an amorphous alloy saves electricity at eddy currents in the electrical industry. Besides, the reduction of losses in the use of such materials and high operating temperature ensures a reduction in the equipment's size.

At present, the study of ferrimagnetic materials is an urgent task, as several important tasks are being solved [5]: First, new devices and devices are being developed that allow solving problems arising in the electric power industry (for example, suppression of high-frequency pulse signals). Secondly, the properties of ferrimagnetic materials themselves are studied, their effect in composite compositions. This is important since new nanocrystalline materials can replace the use of expensive rare-earth metal materials with more affordable, cheap, and effective materials [6], [7].

In the late 80 s of the last century, alloys were developed that, under the action of heat treatment, acquire a nanocrystalline structure [8]. 
The result of the research was a series of alloys with a mixed amorphouscrystalline structure. These alloys have a unique complex of magnetic, primarily hysteresis properties [9]. The characteristics of the alloys provide a high rate of nucleation of crystals while delaying their growth. This makes it possible to obtain an amorphous-crystalline structure due to the annealing of such an alloy. However, until recently, these alloys were used only in the manufacture of transformer equipment.

Now, one of the promising applications of ferromagnetic materials is their use in devices for protection against transient processes, very fast transient overvoltages (VFTO) [10].

\section{Materials}

It was suggested to propose the use of ferromagnetic tape based on nanocrystals (5BDSR), which is produced at the Ashinsky Metallurgical Plant (Russia) by casting a flat stream of liquid metal onto the surface of a cooled drum [11]. It is used in the amorphous or amorphous-nanocrystalline state, for example, for magnetic circuits.

The advantages of the alloy are very high resistance at lightning and switching overvoltage frequencies $(100 \mathrm{kHz}-2 \mathrm{MHz})$, high values of induction at low coercivity, low losses in the reversal at high frequencies, almost zero magnetostriction, high specific resistance, high values of magnetic permeability, which, depending on the method of thermomagnetic processing, can reach values of 50,000. Physical properties and characteristics tapes are presented in the table (Table. 1).

Table 1. The Main Characteristics of The Tape 5BDSR

\begin{tabular}{|c|c|}
\hline Properties & Values \\
\hline Curie Temperature $\mathrm{T \kappa},{ }^{\circ} \mathrm{C}$ & 350 \\
\hline The Temperature of The Onset of Crystallization $\mathrm{TC},{ }^{\circ} \mathrm{C}$ & 530 \\
\hline Density, $\mathrm{g} / \mathrm{cm}^{3}$ & 7,6 \\
\hline Electrical Resistivity, $\mu \Omega \times \mathrm{m}$ & 1,6 \\
\hline Constant Magnetostriction, ppm & 2,1 \\
\hline Maximum Continuous Use Temperature, ${ }^{\circ} \mathrm{C}$ & 240 \\
\hline Bs Saturation Induction, $\mathrm{T}$ & 1,30 \\
\hline
\end{tabular}

\section{Application of Materials}

The properties of the tape allow it to be used to create a frequency-dependent signal. This, for example, allows the development of devices in which the resistance increases with increasing signal frequency. The reason for this is the emerging skin effect: an inhomogeneous distribution of the density of alternating current across the conductor section. This is due to the ejection of primary electric and magnetic fields from the conductor's bulk by secondary induced fields. In this case, the maximum current density values are reached on the surface and decrease with depth. It is easy to estimate 
the depth of the skin layer over which current flows knowing the characteristics of the material and the frequency of the current using the well-known expression (1):

$$
\lambda=\sqrt{\frac{2}{\mu_{0} \cdot \mu \cdot \sigma \cdot \omega}} .
$$

Where: $\mu_{0}-$ the magnetic constant; $\mu$ - the magnetic permeability of the material; $\sigma-$ conductivity; $\omega$ - current frequency.

The traditional skin effect in metals has a weak dependence of resistance on frequency. The maximum frequency dependence is achieved when the thickness of the skin layer is many times smaller than the conductor's transverse dimensions and must correspond to $\sqrt{\omega}_{\omega}$. It can be calculated that, for example, in aluminum, the ratio of resistance at $50 \mathrm{kHz}$ to resistance at $50 \mathrm{~Hz}$ cannot exceed 30 for any homogeneous metals [12].

However, the use of ferromagnetic material in double-layer construction can significantly increase the conductor's resistance when exposed to high frequency signals due to the large decrease in the thickness of the conductive layer in the material. Provided that the skin layer's thickness becomes less than the thickness of the ferromagnetic patch on a conventional electrically conductive metal. In this case, the resistance ratio at frequencies from $50 \mathrm{kHz}$ to $50 \mathrm{~Hz}$ can reach 10,000 or more. This effect can be used to protect substation equipment from high-frequency signals coming from overhead lines (for example, lightning impulses or switching overvoltages). These high-frequency signals can be hazardous for substation equipment.

It is known that thunderstorm overvoltage pulses have a frequency of about 300 $\mathrm{kHz}$. Consequently, when a lightning strike an overhead line (OHL) and an overvoltage pulse is formed, the network's frequency changes from an operating $50 \mathrm{~Hz}$ to a frequency of a lightning pulse $(300 \mathrm{kHz})$. Accordingly, the resistivity of the composite conductor, if aluminum is taken as its basis, under the action of the skin effect will increase from $2.65 \cdot 10^{-}$ ${ }^{8} \mathrm{Ohm} \mathrm{m}$ at a frequency of $50 \mathrm{~Hz}$ to several tens $\mathrm{Ohm} \mathrm{m}$ at a frequency of $300 \mathrm{kHz}$. This will be enough to reduce the overvoltage pulse's amplitude to safe values for the substation equipment.

Previously was developed a device for protection against high-frequency overvoltages using an amorphous nanocrystalline material, based on the use of the arising abnormal skin effect - a frequency-dependent device (FDD) [13]. The ferromagnetic tape used by us is an amorphous nanocrystalline magnetically soft alloy (FeNbCuCoBSi), which has a very high magnetic permeability [14].

The device is a three-layer cable, consisting of a conductive wire with a layer of ferromagnetic material deposited on it; the cable is coated with a high-voltage dielectric material from the outside. The cable itself is wound into a coil of dielectric material (Figure 1). At an operating frequency of $50 \mathrm{~Hz}$, the device's resistance corresponds to the resistance of the phase conductor. However, when passing, for example, a lightning surge having a frequency range of $200-300 \mathrm{kHz}$, due to the displacement of the current into the ferromagnetic layer, the device's resistance increases sharply to 150-200 Ohms, which reduces the pulse amplitude. Besides, the device's high inductance makes it possible to smooth the pulse, making it safe for transformer equipment. 


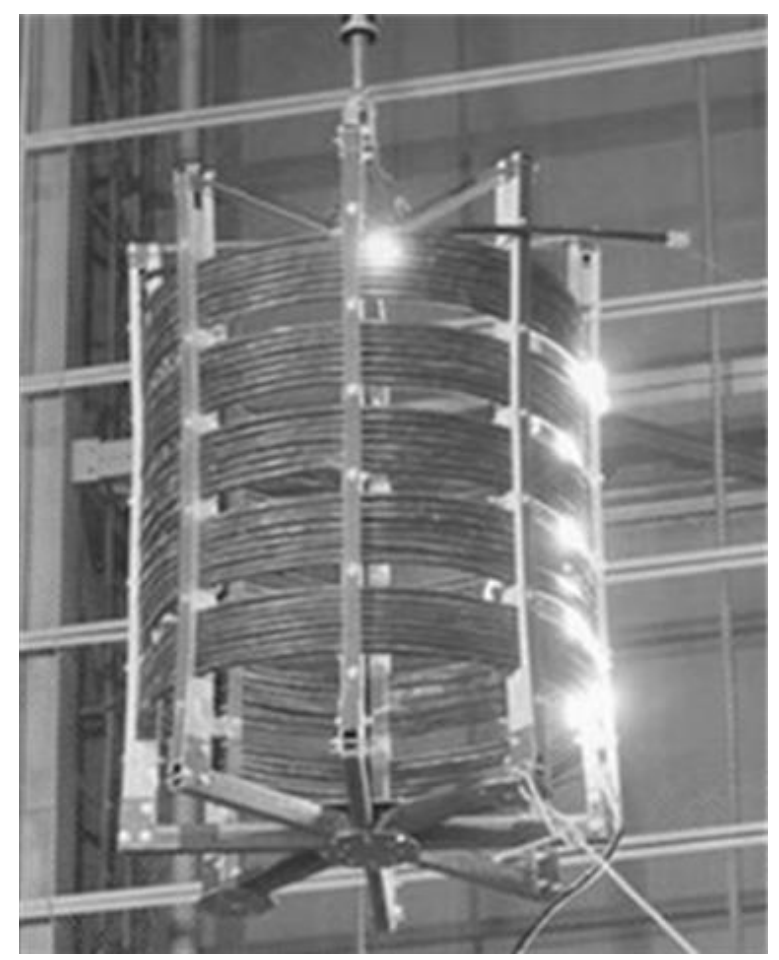

Figure 1. The FDD is tested in the high-voltage hall

The success achieved in the application of ferromagnetic materials has allowed our department to work on improving FDD. One of the main areas of further development is the development of composite multilayer cables of various shapes. Thus, to increase the active resistance and, consequently, to reduce the overall dimensions of the device, instead of conductive aluminum wire, a conductor is examined in the form of a "sandwich" made of parallel-connected conductive plates (aluminum, copper) and a ferromagnetic tape (Figure 2).

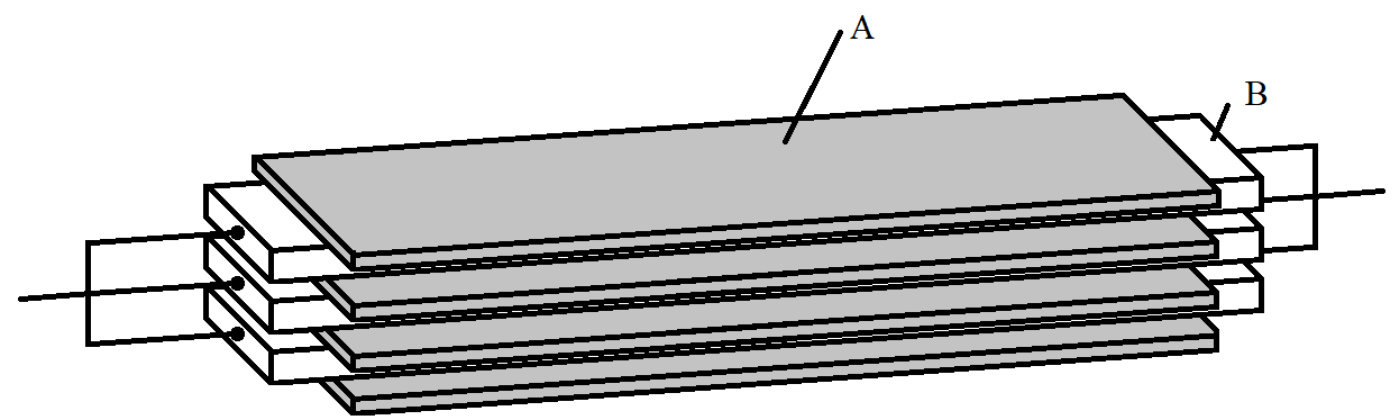

Figure 2. Schematic diagram of a flat conductor FDD: A - Ferromagnetic tape; B Conductive material

The prototypes for testing consisted of a round FDD wire and a flat multilayer FDD wire. Round wire FDD is a three-layer coaxial wire (Figure 3). The inner layer is represented by aluminum wire A-95, with a cross-sectional area of $95 \mathrm{~mm}^{2}$ (this crosssectional area is used in Russia for $35 \mathrm{kV}$ overhead lines), a layer of ferromagnetic tape 0.3 $\mathrm{mm}$ thick is applied to it. The third, outer layer is the TUT heat-shrinkable tube, which protects the ferromagnetic layer from its external mechanical and climatic influences. 


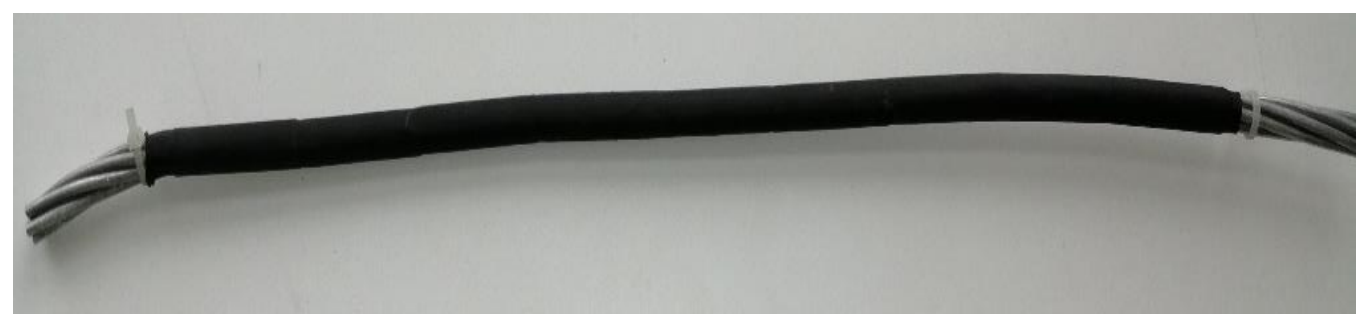

a)

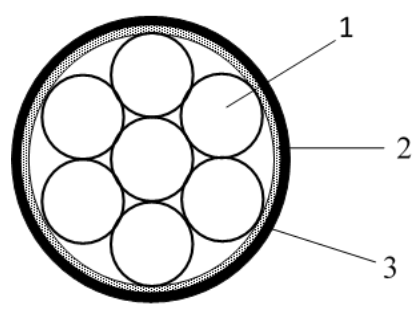

b)

Figure 3. The round wire sample a) the photo of a sample, b) the diagram of a wire structure: 1) an aluminum conductor, 2) a ferromagnetic layer, 3) a dielectric protective layer.

The flat sample shown in Figure 4 consisted of 6 layers of an aluminum conductor, alternating with seven layers of a 5BDSR ferromagnetic tape. Each layer of the conductor was $0.3 \mathrm{~mm}$ thick and $50 \mathrm{~mm}$ wide, the thickness of the ferromagnetic layers was also, $0.3 \mathrm{~mm}$, and the width was $60 \mathrm{~mm}$. Due to the different widths, a protrusion of the ferromagnetic material was provided along the edges by $5 \mathrm{~mm}$, on each side. This should increase efficiency by injecting current into the thin ferromagnetic layer. So far, compression layers have been used to maintain the shape. In the future, it is planned to replace it with a more convenient and ergonomic dielectric design.

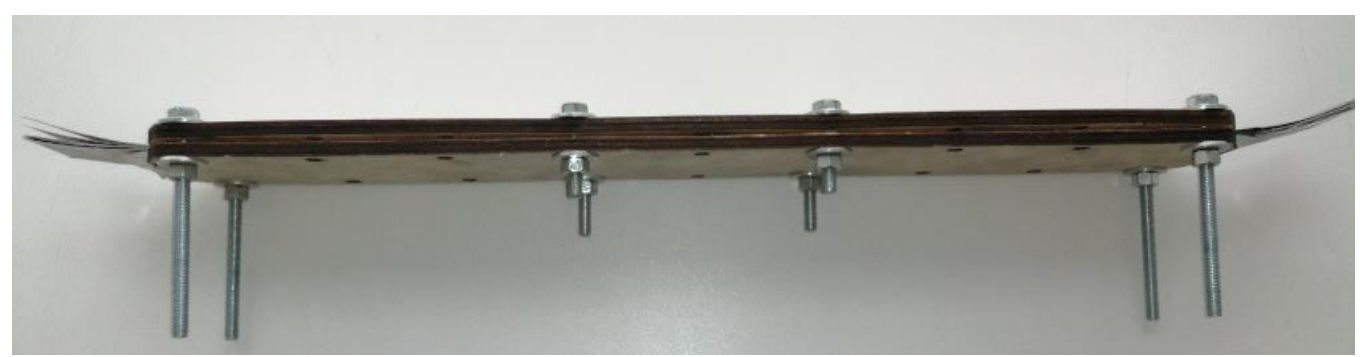

a)

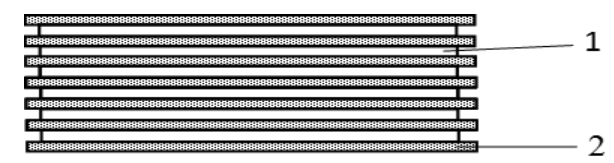

b)

Figure 4. The flat wire sample: a) a photo of a sample, b) a diagram of the wire construction: 1) an aluminum conductor, 2) a ferromagnetic layer. 
Both wires had an aluminum conductive part, were 0.7 meters long. The crosssectional area of the conductor in both layouts was the same and amounted to $95 \mathrm{~mm}^{2}$.

The tests used a two-channel oscilloscope DS1022C. This oscillograph makes it possible to determine the total complex resistance of the layout. To do this was compared the rms voltages taken by the K1 and K2 channels of the oscilloscope from the R2 (testing sample) layout and the low-inductance resistance $\mathrm{R} 1$ with a known active resistance value $(0,01 \mathrm{OM})$. As a source of high-frequency signals, the SFG-2010 generator was used (Figure 5).

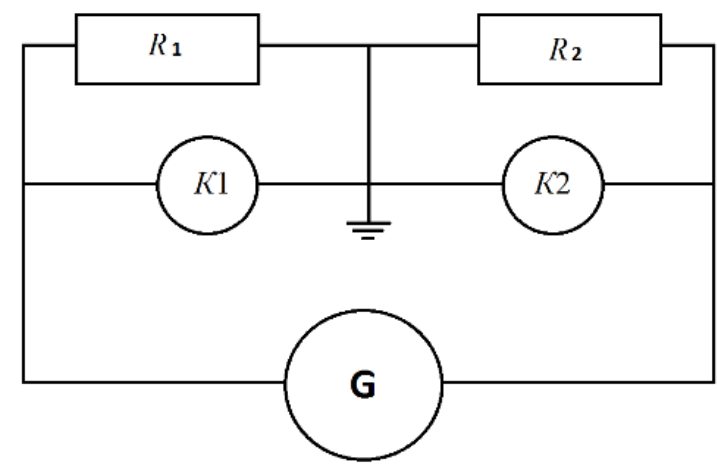

Figure 5. Measurement scheme

On the oscilloscope screen in the cursor measurement mode, the repetition periods of the signals and the phase shift between the signal amplitudes was determined. These signals were taken from the layout and shunt resistance. The values of reactive $X$ and active $\mathrm{R}$ resistance of the sample were calculated from the obtained values of the total complex resistance $\mathrm{Z}$ and the phase shift $\varphi$. The resulting resistance was compared with the sample's resistance at a frequency of $50 \mathrm{~Hz}$ and measured with an impedance meter.

Comparing the effectiveness of samples of a conductor of circular cross-section and a multilayer conductor showed that the latter shows a higher increase in active resistance with increasing signal frequency. Figure 6 shows that at thunderstorm frequencies $(200-300 \mathrm{kHz})$, a multilayer sample's efficiency is $20-30 \%$ higher. At switching overvoltage frequencies $(1 \mathrm{MHz})$, the efficiency is more than two times compared with a circular sample.

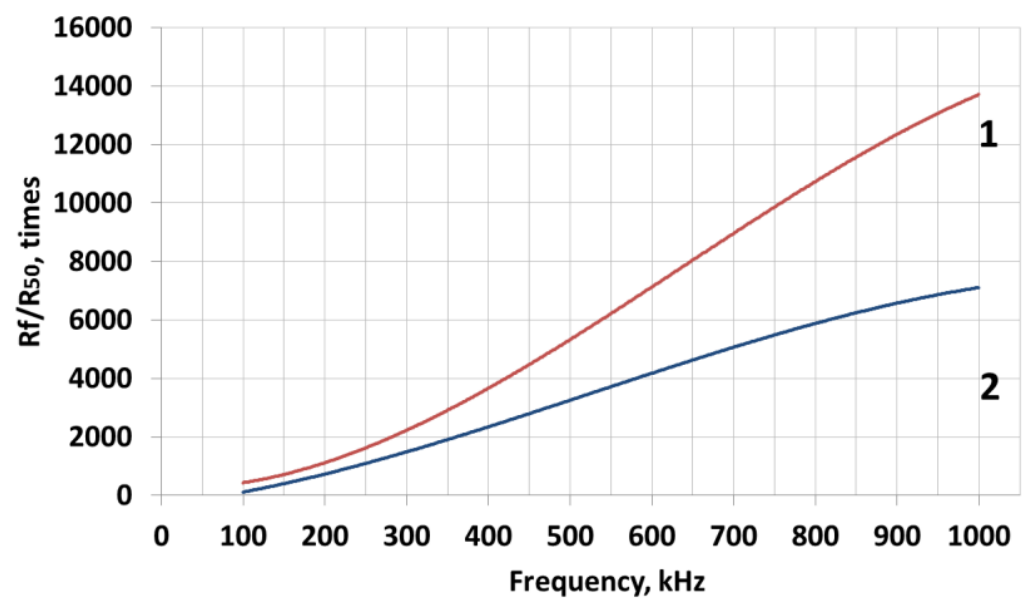

Figure 6. Test results: 1 - multilayered conductor; 2 - round conductor 


\section{Results}

High efficiency of using composite cables using ferromagnetic materials is shown by both laboratory tests and three-year monitoring of the operation of three FDD prototypes installed in 2014 at substation $110 \mathrm{kV}$ Sugmutskaya of Tyumenenergo OJSC. The monitoring system continuously monitors the input and output voltages of the device. For three thunderstorm periods, quite many overvoltage pulses of various amplitudes and different origins, which came to the substation with overhead lines, were recorded. Figure 7 shows two typical recorded overvoltage events for 2017. The use of a frequencydependent device made it possible to reduce the amplitudes of the incoming pulses significantly. The amplitude of some of them was about $1 \mathrm{MV}$. However, the interference either was suppressed at the frequency-dependent device's output, or they were already within acceptable limits.
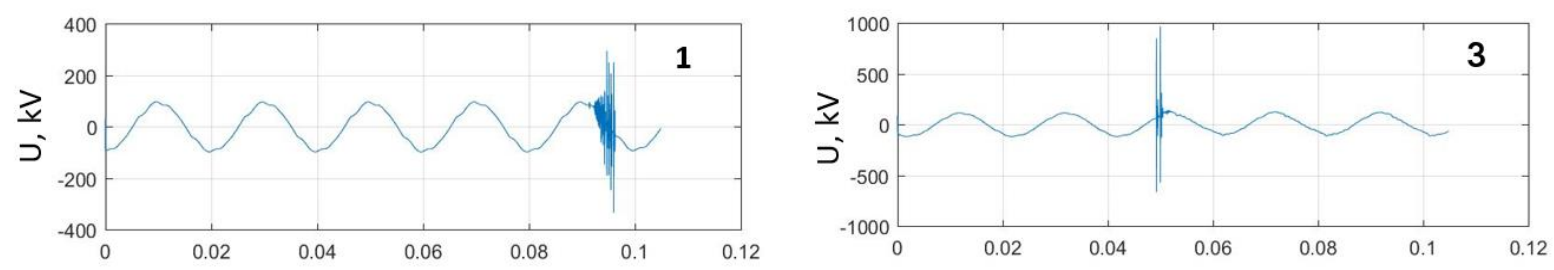

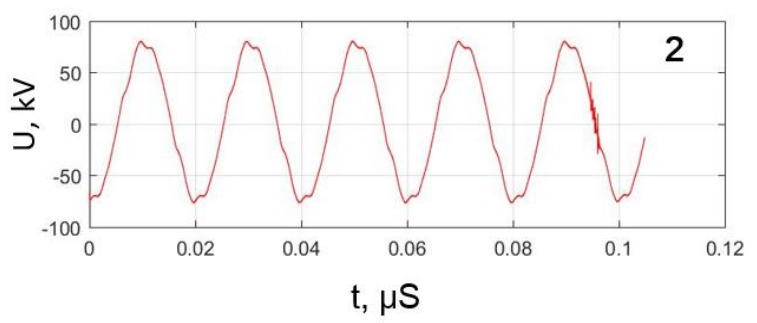

A)

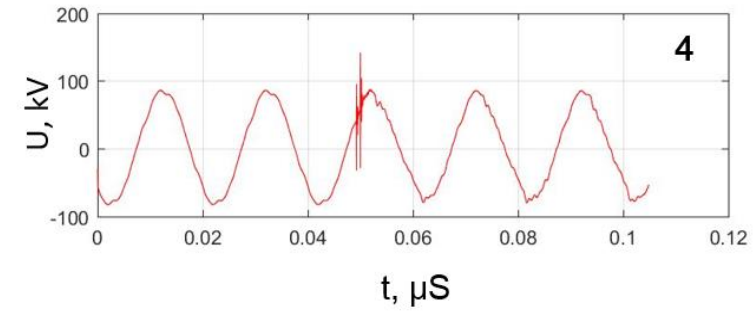

B)

Figure 7. Monitoring results: A, B - overvoltage pulses 18.05.2017 and 02.06.2017; 1, 3 Signal at the input to the device; 2, 4- output signal from the device

\section{Conclusions}

The use of ferromagnetic nanocrystalline materials and composite structures based on them can significantly increase the efficiency and safety of the equipment being developed in the energy field. One of the most promising areas of applying of these materials is the protection of equipment from high-frequency overvoltages. For example, a two-layer combination of a conductor and ferromagnetic material can be effectively used to protect electrical equipment from high-frequency overvoltages.

Thanks to this, there is an opportunity to increase the efficiency of a frequencydependent device significantly. For example, making a conductive cable in the form of a flat frequency-dependent resistor, which consists of plates of conductive and ferromagnetic material.

The flat conductor has a much more excellent active resistance than that of cylindrical samples. It also has much smaller dimensions and weight, which will significantly facilitate its installation and operation at the facilities. 


\section{References}

[1] R. Kolano, A. Kolano-Burian, M. Polak, and J. Szynowski, "Application of rapidly quenched soft magnetic materials in energy-saving electric equipment," IEEE Transactions on Magnetics, Vol. 50, No. 4, pp. 1-4, 2014. doi: 10.1109/TMAG.2013.2283918

[2] J. Petzold, "Applications of nanocrystallines of magnetic materials for modern electronic devices," Scripta Materialia, Vol. 48, No. 7, pp. 895-901, 2003. doi: 10.1016/S1359-6462(02)00624-3

[3] A.M. Leary, P.R. Ohodnicki, and M.E. Mchenry, "Soft magnetic materials in highfrequency high-power conversion applications," Journal of Metals, Vol. 64, No. 7, pp. 772-781, 2012. doi: 10.1007/s11837-012-0350-0

[4] P. Marin, A.M. Aragon, V. Lopez, and A. Hernando, "Nanocrystalline soft magnetic ribbons and microwires: Towards future developments in energy related applications," Paper presented at IEEE International Conference on Computer as a Tool, 2015, Salamanca, Spain, doi: 10.1109/EUROCON.2015.7313745

[5] M. Kachniarz, and D. Jackiewicz, "Study on the magnetizing frequency dependence of magnetic characteristics and power losses in the ferromagnetic materials," In: Recent Global Research and Education: Technological Challenges Proceedings of the $15^{\text {th }}$ International Conference on Global Research and Education Inter-Academia, Warsaw, Poland, 2015. doi: 10.1007/978-3-319-46490-9_6

[6] L.H. Lewis, and F. Jiménez-Villacorta, "Perspectives on permanent magnetic materials for energy conversion and power generation," Metallurgical and Materials Transactions A, Vol. 44, pp. 2-20, 2013. doi: 10.1007/s11661-012-1278-2

[7] I.D. Ibrahim, E.R. Sadiku, T. Jamiru, Y. Hamam, Y. Alayli, and A.A. Eze, "Prospects of nanostructured composite materials for energy harvesting and storage," Journal of King Saud University - Science, Vol. 32, No. 1, pp. 758-764, 2020. doi: 10.1016/j.jksus.2019.01.006

[8] V.E. Roshchin, and A.V. Roshchin, Basics of Production of Nanocrystalline and Amorphous Metals, Center SUSU, Chelyabinsk, Russia, 2009.

[9] M.R. Filatov, Yu.A. Anikin, Yu.B. Levin, Theoretical Basis for the Production of Amorphous and Nanocrystalline Alloys by the Method of Ultrafast Quenching, Moscow MISIS, Moscow, Russia, 2006.

[10] X. Zhang, and J. He, "Lightning overvoltage suppression of power transformer in UHV GIS by ferromagnetic ring," Paper presented at 7th Asia-Pacific International Conference on Lightning, 2011, Chengdu, China, doi: 10.1109/APL.2011.6110197

[11]Asha Metallurgical Plant, " $5 B D S R$ Brand Tape" (n.d.) [Online]. Available at: https://www.amet.ru/buyers/product/tape/24/ [Accessed: June 2020]

[12] S.M. Korobeynikov, A.P. Drozhzhin, and L.I. Sarin, "The skin effect in composite materials," Electricity, Vol. 7, pp. 2-9, 2004 (In Russian).

[13] S.M. Korobeynikov, and N.Ya. Ilyushov, "High-current testing of frequency dependent device," Paper presented at The $11^{\text {th }}$ International Forum on Strategic Technology, Novosibirsk, Russia, 2016, doi: 10.1109/IFOST.2016.7884260

[14] M. Ohnuma, K. Hono, S. Linderoth, J.S. Pedersen, Y. Yoshizawa, and H. Onodera, "Small-angle neutron scattering and differential scanning calorimetry studies on the copper clustering stage of $\mathrm{Fe}-\mathrm{Si}-\mathrm{B}-\mathrm{Nb}-\mathrm{Cu}$ nanocrystalline alloys," Acta Materialia, Vol. 48, pp. 4783-4790, 2000. doi: 10.1016/S1359-6454(00)00277-9 